\title{
Treatment Following an Evidence-Based Algorithm versus Individualised Symptom-Oriented Treatment for Atopic Eczema
}

\author{
A Randomised Controlled Trial \\ Jochen Schmitt ${ }^{\mathrm{a}} \quad$ Michael Meurer $^{\mathrm{a}} \quad$ Uta Schwanebeck $^{\mathrm{b}} \quad$ Xina Grählert $^{\mathrm{b}}$ \\ Knut Schäkel ${ }^{a}$ \\ a Department of Dermatology, University Hospital Carl Gustav Carus, and ${ }^{b}$ Coordination Centre for Clinical Studies, \\ Medical Faculty Carl Gustav Carus, Technical University Dresden, Dresden, Germany
}

\section{Key Words}

Atopic dermatitis · Atopic eczema $\cdot$ Long-term control •

Randomized controlled trial · Topical calcineurin inhibitors •

Topical corticosteroids $\cdot$ Treatment algorithm

\begin{abstract}
Background: Evidence-based treatment algorithms, successfully established for asthma, are missing for atopic eczema (AE). Objectives: To investigate whether treatment according to an evidence-based algorithm is an effective and applicable concept for the management of AE. Methods: Based on a systematic literature review, we developed an evidence-based severity-score-oriented treatment algorithm for $A E$ and compared its effectiveness to that of an individualised symptom-oriented treatment (individual therapy) in a randomised controlled trial. Sixty-three participants were randomised to algorithm $(n=32)$ or individual therapy $(n=31)$ and treated accordingly for 12 months. Study end points included difference between baseline SCORAD and mean SCORAD under treatment (primary end point), quality of life and treatment utilisation. Analysis was by intention to treat (registration: ClinicalTrials.gov:NCT00148746). Results: No statistically significant differences in clinical or subjective response were observed between groups. Treatment following the algorithm and individual treatment both effectively controlled AE. Mean SCORAD reductions were $47 \%$
\end{abstract}

(95\% confidence interval, $\mathrm{Cl}=38-55$; algorithm) and $42 \%$ ( $95 \% \mathrm{Cl}=29-54$; individual). Clinical response was paralleled by improved quality of life in both groups. Physicians adhered to the algorithm option in $93 \%$ of their treatment decisions. Conclusion: Treatment following an evidence-based algorithm is an effective and applicable concept for the management of $\mathrm{AE}$ but does not show clear advantages compared to individualised treatment in a dermatological setting.

Copyright $\odot 2008$ S. Karger AG, Basel

\section{Introduction}

Atopic eczema (AE, synonymous with atopic dermatitis) is a multifactorial, itchy, inflammatory skin disease that affects up to $20 \%$ of children and $2-4 \%$ of adults [1, 2]. Even patients with clinically mild disease frequently report substantial problems in everyday life [3]. AE imposes a high economic burden with both total cost and out-of-pocket expenses similar to those of asthma [4].

M.M. has served as a consultant for Novartis, Wyeth and Biotest, and as a paid lecturer for Novartis, Wyeth, Biotest, Leo, Actelion, $3 \mathrm{M}$ and ZLB Behring. K.S. has served as a paid lecturer for Novartis, ALK-Scherax and Bencard.

\section{KARGER}

Fax +41613061234 E-Mail karger@karger.ch www.karger.com
(C) 2008 S. Karger AG, Basel

$1018-8665 / 08 / 2174-0299 \$ 24.50 / 0$

Accessible online at:

www.karger.com/drm
Jochen Schmitt, MD, MPH

Department of Dermatology, University Hospital Carl Gustav Carus

Technical University Dresden, Fetscherstrasse 74, DE-01307 Dresden (Germany)

Tel. +49 351458 3860, Fax +493514585326

E-Mail jochen.schmitt@uniklinikum-dresden.de 
Currently not curable, the symptomatic treatment includes topical anti-inflammatory substances such as topical corticosteroids and topical calcineurin inhibitors, phototherapy, and systemic immunosuppressive agents such as oral corticosteroids, cyclosporine and azathioprine [5]. Only few head-to-head trials have been published for AE [5-7]. Drugs assessed in different placebocontrolled trials are not objectively comparable because of heterogeneous inclusion criteria and outcomes used [8]. Although some of the published treatment guidelines for AE have been developed using evidence-based methods, they have not been validated using methods of evidence-based medicine, i.e. by applying them in the setting of a randomised controlled trial (RCT) [9-11].

For other chronic conditions such as allergic asthma, chronic pain or depression, it has been shown that severity-score-based treatment algorithms improve disease management and control [12-14]. Recent studies suggest that in children with AE early and sustained disease control may decrease the risk of subsequent atopic diseases including allergic rhinitis and asthma $[15,16]$. Furthermore, in both children and adults effective control of inflammation seems to decrease the risk of persistent disease and may thus modify the course of $\mathrm{AE}[17,18]$. A validated treatment algorithm may therefore help to improve medical care of patients with AE.

We developed a standardised, evidence-based, timeand severity-score-dependent treatment algorithm for children and adults with moderate-to-severe AE ('algorithm') that gives explicit recommendations for objectively defined disease states and tested its effectiveness against individualised symptom-oriented treatment ('individual') in a pragmatic RCT.

\section{Methods}

\section{Development of the Time- and Severity Score-Oriented} Treatment Algorithm

A comprehensive literature review was conducted using multiple overlapping search strategies to develop an evidence-based treatment algorithm for moderate-to-severe AE that gives exact recommendations including name of drug, frequency and duration to be used depending on objective disease severity, prior course of disease and patient age. We searched Medline and the Cochrane central register of controlled trials (both from inception until December 2003) using different combinations of the medical subject $(\mathrm{MeSH})$ terms 'atopic dermatitis', 'randomised controlled trial', 'review', 'guideline', 'consensus' and text key words including the names of all drugs approved for AE. To identify additional articles with potential information we also reviewed the bibliographies of selected key articles [6, 9, 19-21]. Finally, we reviewed the summary of product characteristics of all treatment options for moderate-to-severe AE that we intended to include in the treatment algorithm based on our literature search.

We only identified few head-to-head RCTs [22-28]. The inclusion criteria and outcomes used in the placebo-controlled trials are too heterogeneous to objectively compare interventions across trials $[5,6,29,30]$. The treatment guidelines identified suggest multiple drug classes (e.g. topical corticosteroids, topical calcineurin inhibitors) for not objectively defined disease states (e.g. severe refractory disease, acute exacerbation, persistent disease, flare). However, guidelines do neither specify exactly which drug to administer for how long in a certain disease state nor do they objectively define disease states by explicit criteria (e.g. disease severity score, quantitative definition of 'persistent disease') $[9,11]$.

Therefore, it was not possible to base our study on an algorithm that is definitely the most effective according to the published evidence. Our goal was to develop an algorithm that combines the evidence of RCTs with the regulatory guidelines (summary of product characteristics) of the drugs used and thus to include everyday clinical practice into the setting of a clinical trial

The treatment algorithm was drafted by J.S., revised by M.M. and K.S., and finalised by consensus of all authors.

The final algorithm consists of two steps: (1) stepwise treatment escalation to induce response (fig. 1a); (2) management of flares depending on previously effective treatment (fig. 1b).

\section{Study Design and Participants}

We performed a pragmatic, investigator-initiated, parallelgroup, randomised, patient-blinded, active controlled clinical trial to assess the effectiveness and safety of the time- and severityscore-dependent treatment algorithm ('algorithm') compared to individualised symptom-oriented treatment ('individual'). The study protocol was approved by the local ethics committee. All study subjects gave written informed consent before trial participation. No external sponsor was involved in this investigator-initiated study. The study was registered at ClinicalTrials.gov (identifier: NCT00148746).

The study was conducted at the outpatient clinic of the Department of Dermatology, Medical Faculty Carl Gustav Carus, University of Technology, Dresden, Germany. Between June 2004 and September 2005, 64 patients aged 2 years and older with AE [31], who had at least moderate disease activity with a SCORAD [32] (scoring atopic dermatitis) score $\geq 20$, were enrolled. Patients with prior malignant disease, women without adequate contraception and pregnant/nursing women were not eligible. There was no wash-out phase for any drugs administered prior to inclusion. Participants were mainly recruited by referrals from dermatologists and paediatricians, and by information about this study in the local press.

Six dermatologists were randomly allocated at a ratio of $1: 1$ by drawing identically looking lots to treat patients either strictly following the algorithm or to perform individualised symptom-oriented treatment (individual). During the screening visit, patients underwent central randomisation with the use of a permutatedblock randomisation list (block length 4), with equal allocation to the algorithm and individual groups. The allocation sequence was generated by SPSS (version 11.5.1, SPSS Inc.). Stratification by disease severity (SCORAD 20-50 vs. SCORAD > 50) and by par- 
Fig. 1. Time- and severity-score-dependent treatment algorithm for moderateto-severe AE with stepwise treatment escalation to induce response (a) and management of flares depending on previously effective treatment (b). Moderate AE: baseline SCORAD 20-50; severe AE: baseline SCORAD >50. Tacrolimus: age 2-15 years, $0.03 \%$; 16 years or older, $0.1 \%$. Topical steroids: face, hydrocortisone $1.0 \%$; body, with SCORAD $>50$ mometasone furoate $0.1 \%$, with SCORAD $\leq 50$ prednicarbate $0.25 \%$; dose: week 1 b.i.d., week 2-3 once a day, week 4 every other day. Prednisolone: days $1-3,1.0 \mathrm{mg} / \mathrm{kg}$ BW; days $4-7,0.5 \mathrm{mg} / \mathrm{kg} \mathrm{BW}$; days $8-10,0.25$ $\mathrm{mg} / \mathrm{kg}$ BW; days 11-14, $0.1 \mathrm{mg} / \mathrm{kg}$ BW. Topical calcineurin inhibitors (TCI): pimecrolimus b.i.d. (face); tacrolimus b.i.d. (body) until 1 week after remission (defined as SCORAD <8); repeated application as soon as first clinical signs or symptoms of eczema appear. Response defined as SCORAD 50, i.e. relative reduction compared to baseline SCORAD $\geq 50 \%$. Cyclosporine: initial dose, $5 \mathrm{mg} / \mathrm{kg}$ BW; stepwise reduction to minimum effective dose; minimum treatment duration 6 weeks (in the absence of adverse reactions); treatment may be continued up to 1 year. Flare: increase in SCORAD to a score $>50 \%$ of baseline SCORAD after prior response.
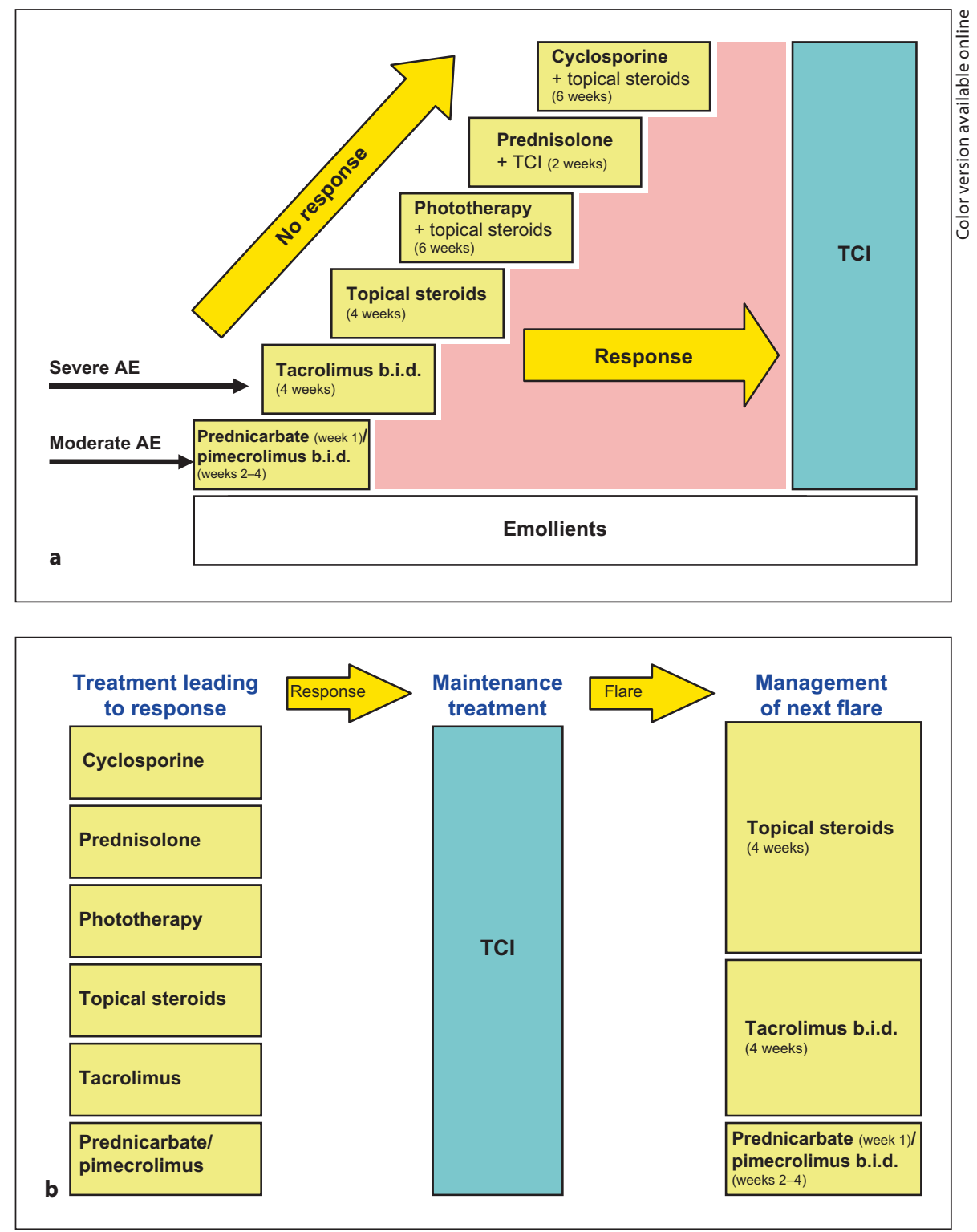

ticipant age ( $2-15$ vs. $\geq 16$ years) was used to minimise baseline differences in these potential confounders. The allocation sequence was concealed to all investigators until interventions were assigned. Throughout the study the participants did not know which treatment had been allocated.

\section{Study Procedures}

In both study groups patients were treated exclusively with drugs approved for AE. All drugs were supplied by prescription. Additional use of emollients was permitted, and patients on antihistamines at baseline were allowed to continue on the same dose throughout the study. Physicians allocated to follow the algorithm were only allowed to violate the treatment scheme (fig. 1) if a proposed drug was contra-indicated. At the end of the study, one investigator (J.S.) double-checked the treatments prescribed to the participants allocated to the algorithm group and recorded all violations against the treatment scheme.

Study visits were scheduled every 4 weeks ( \pm 1 week) for a total of 1 year (13 visits after baseline). At each visit we assessed: current objective disease activity by means of the SCORAD, current global disease severity independently assessed by the physician (investigator global assessment, IGA [33]) and by the patient (patient global assessment, PGA), and the current impact of AE on everyday life using the children [34] or adult [35] version of the dermatology life quality index (DLQI; children: 5-16 years; adults: 17 years or older). Quality of life was not assessed in children aged $<5$ years.

The primary measure of effectiveness was the difference between baseline SCORAD and mean SCORAD under treatment. All other variables were defined as secondary end points. The SCORAD is the most widely used and most extensively validated 
severity index for AE [8]. The SCORAD combines an assessment of disease extent using the rule of nines with 6 typical clinical features of disease intensity (erythema, oedema/papulation, oozing/ crusts, excoriations, lichenification; each assessed on a 4-point Likert scale at a single representative site) plus a $10-\mathrm{cm}$ visual analogue scale for itch and sleep loss due to AE. The SCORAD ranges from 0 to 103 with higher scores indicating severer disease [32]. Severe AE was defined as SCORAD $>50$, moderate AE as SCORAD 20-50. Response was defined as a relative improvement in total SCORAD by $50 \%$ or more compared to baseline (SCORAD 50). Remission was defined as SCORAD $<8$ [30, 36-38]. SCORAD training was attended by all assessors prior to study initiation [32, 37].

The IGA and PGA are static instruments to assess global disease severity on a 6-point Likert scale by the physician (IGA) and patient (PGA), respectively [33]. DLQI and Children's DLQI are widely used instruments with adequate psychometric properties to measure health-related quality of life in dermatological patients. The (Children's) DLQI score ranges from 0 to 30 with higher scores (over 10) reflecting worse quality of life [34, 35, 39, 40]. Adverse events were recorded according to the Common Toxicity Criteria for Adverse Events, version 3.0 [41].

\section{Statistical Analysis}

The primary end point was the difference in absolute change in SCORAD between baseline and mean SCORAD under treatment. We aimed to detect an 8-unit difference between groups. On the assumption of a standard deviation of 10 units in both groups, calculations showed that 52 participants were needed for a study with $80 \%$ power and $5 \%$ significance (nQuery-Advisor ${ }^{\circledR} 4.0$ ). We aimed to recruit 64 patients to allow for a $20 \%$ drop-out rate.

As secondary end points, we analysed proportions of participants with remission (SCORAD $<8$ ), with response (SCORAD 50 ), with significant benefit in quality of life (DLQI change $\geq 5$ units) [40] and with a lower global disease severity score as assessed by the physician (IGA) and patient (PGA) at any study visit after baseline and at more than $50 \%(n=7)$ study visits, respectively. For the latter analysis we assumed that a criterion (e.g. response) was not met if a participant did not attend a study visit.

Additionally, we compared the proportions of participants receiving and the average amount of anti-inflammatory drugs prescribed for AE by drug group, with glucocorticosteroids being classified according to Niedner [42].

Primary data analysis was by intention to treat and included all randomised participants who attended the baseline visit. In the per-protocol analysis (secondary analysis) we included all participants who attended at least $80 \%(n=12)$ of all study visits. Missing data were not replaced. Proportions were compared using the $\chi^{2}$ test or Fisher's exact test, continuous variables by t test or Mann-Whitney test, as appropriate [43]. Data were analysed with SPSS, version 11.5.1.

\section{Results}

Figure 2 shows the trial profile. Sixty-four patients (all Caucasians born in Germany) were enrolled and randomly allocated to the algorithm $(\mathrm{n}=32)$ and individual groups $(\mathrm{n}=32)$. One patient allocated to individual therapy withdrew before baseline. The following results are based on the 63 participants who attended the baseline visit (fig. 2). Demographic data and disease characteristics were balanced between groups (table 1). A total of 20 children aged 2-15 years were included in this study, 10 of whom were allocated to each treatment group. The mean SCORAD at baseline was 46 units in both groups.

There was no statistically significant difference in mean SCORAD change between algorithm and individual groups. Treatment was highly effective in both groups with mean reductions in disease activity of 21.4 SCORAD units (95\% confidence interval, $\mathrm{CI}=17.6-25.2$; i.e. $47 \%, 95 \% \mathrm{CI}=38-55)$ by algorithm $(\mathrm{p}<0.001)$, and 19.3 SCORAD units $(95 \% \mathrm{CI}=13.6-24.9 ; 42 \%, 95 \% \mathrm{CI}=29$ 54) by individual therapy ( $<<0.001$; table 2$)$.

Figure 3 shows the course of mean (with 95\% CI) disease activity (SCORAD) from baseline over the 1-year study period. Compared to baseline, mean disease activity was significantly lower at all following assessments in both groups (fig. 3).

Clinical response was paralleled with improved quality of life (DLQI) in both groups. Mean relative improvement in DLQI was $42 \%(95 \% \mathrm{CI}=19-66)$ in patients allocated to the algorithm group compared to $47 \%$ (95\% $\mathrm{CI}=27-68)$ in those allocated to individual therapy. Results of all secondary outcomes are detailed in table 2 .

For all outcome variables, the conclusions to be drawn from the per-protocol population (fig. 2) were identical with the presented intention-to-treat analysis.

Significantly more patients allocated to algorithm therapy received pimecrolimus $(\mathrm{p}=0.002)$ and tacrolimus $0.03 \%(\mathrm{p}<0.001)$, whereas high-potency corticosteroids (class III) were given significantly more often to patients allocated to individual treatment ( $\mathrm{p}=0.001$; table 3). The trend in treatment utilisation was similar in children and adults, but differences in treatment utilisation were less pronounced in children (tables 3, 4). Despite high baseline severity, phototherapy and systemic treatment were rarely administered in both groups (table 3).

A total of 25 violations against the treatment algorithm were documented, with a maximum of 3 violations per patient. No violations were noted in $14 / 32$ patients allocated to the algorithm group. This implies that physicians adhered to algorithm in $93 \%$ of their treatment decisions (348 visits in the algorithm group, 25 algorithm violations). Adherence to the algorithm was similar in children (94\%) and adults (92\%). In all cases of 


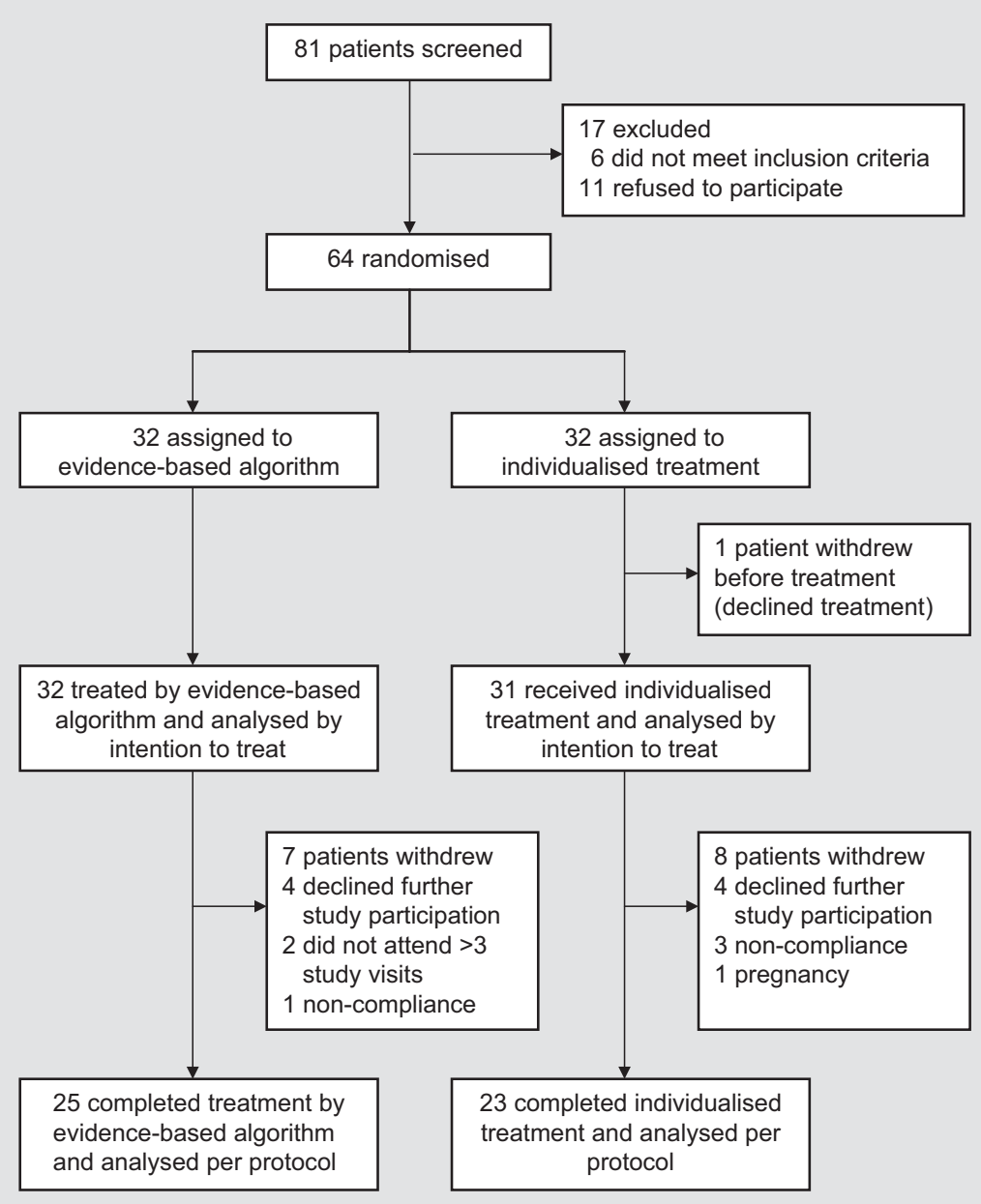

Fig. 2. Trial profile.

violations, corticosteroids were used instead of calcineurin inhibitors.

Adverse events were generally mild with common cold being the most frequently reported condition. No participants developed serious abnormalities in laboratory measures. A total of 8 skin infections [verruca vulgaris $(\mathrm{n}=3)$, tinea $(\mathrm{n}=3)$, folliculitis $(\mathrm{n}=1)$, herpes simplex $(\mathrm{n}=1)$ ] were noted (algorithm $\mathrm{n}=5$, individual $\mathrm{n}=3$ ), all of which were classified as mild. Six serious adverse events were recorded in patients allocated to individual therapy [exacerbation of $\mathrm{AE}(\mathrm{n}=2)$, corneal infection $(\mathrm{n}=1)$, appendicitis $(\mathrm{n}=1)$, traumatic rupture of finger tendon $(\mathrm{n}=1)$, varicosis surgery $(\mathrm{n}=1)$, each requiring hospitalisation] and 1 serious adverse event in patients receiving algorithm treatment (renal colic requiring hospitalisation). All serious adverse events were resolved by the end of study.

Validation of Evidence-Based Treatment Algorithm for Atopic Eczema

\section{Discussion}

This study has two major implications: firstly, it does not show any clear advantage of an algorithm-based treatment concept for moderate-to-severe AE compared to individualised treatment. Secondly, our results demonstrate that disease activity in children and adults with moderate-to-severe AE can be effectively controlled when applying an evidence-based treatment algorithm that considers current disease activity, prior course of disease and the patient's age. Therefore, the concept 'treatment algorithm' appears to be a possible alternative to the current clinical practice of individualised treatment.

Treatment according to a time- and severity-score-dependent algorithm and individualised treatment were both highly effective with mean reductions in disease activity over the 12 -month study period by $47 \%$ in patients

Dermatology 2008;217:299-308 


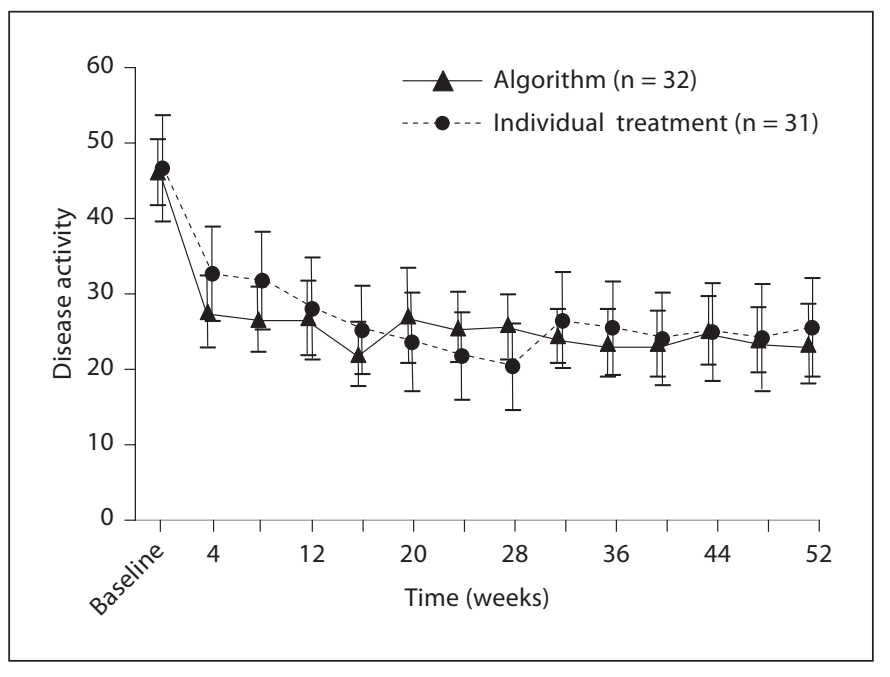

Fig. 3. Disease activity (SCORAD; mean, 95\% CI) from baseline until 52 weeks of follow-up.

allocated to algorithm therapy and $42 \%$ in patients allocated to individual treatment. In both study groups clinical improvements were translated into improved wellbeing as indicated by reduced DLQI scores. No statistically significant differences in clinical or subjective response were observed between groups.

Our study objective was to investigate the effectiveness and applicability of the concept 'evidence-based severityscore-oriented treatment algorithm' in the management of moderate-to-severe AE. Therefore, it was not our goal to develop 'the best' algorithm, but a severity-score-oriented and applicable treatment concept that combines the evidence of RCTs with the regulatory guidelines (summary of product characteristics). Due to studies and new regulatory guidelines (i.e. FDA black box warning for topical calcineurin inhibitors, January 2006) published after the tested algorithm had been developed, the algorithm applied in this study needs to be modified before it can be recommended for current clinical practice [44-47].

There are at least 3 distinct differences between individual symptom-oriented treatment in this trial and management of moderate-to-severe $\mathrm{AE}$ in everyday clinical practice, all of which are potential explanations for the observed benefit in patients allocated to individual treatment. Firstly, the frequency of medical consultation with 14 scheduled appointments within 1 year clearly exceeds common practice in an outpatient setting. It has been suggested that treatment adherence is higher if patients have regular and frequent appointments with their physician
Table 1. Demographics and clinical characteristics of study participants

\begin{tabular}{|c|c|c|}
\hline & $\begin{array}{l}\text { Time- and } \\
\text { severity-score- } \\
\text { dependent } \\
\text { treatment algo- } \\
\text { rithm }(\mathrm{n}=32)\end{array}$ & $\begin{array}{l}\text { Individualised } \\
\text { symptom- } \\
\text { oriented } \\
\text { treatment } \\
(\mathrm{n}=31)\end{array}$ \\
\hline Age, years & $24 \pm 17$ & $21 \pm 13$ \\
\hline Female sex & $18(56)$ & $14(45)$ \\
\hline Allergic rhinoconjunctivitis & $19(59)$ & $21(68)$ \\
\hline Allergic asthma & $10(31)$ & $9(29)$ \\
\hline Raised serum IgE & $28(88)$ & $28(90)$ \\
\hline \multicolumn{3}{|c|}{ Previous treatment for atopic eczema } \\
\hline Topical glucocorticosteroids & $29(91)$ & $31(100)$ \\
\hline Topical pimecrolimus & $21(66)$ & $20(65)$ \\
\hline Topical tacrolimus & $17(53)$ & $16(52)$ \\
\hline Phototherapy & $10(31)$ & $12(39)$ \\
\hline Systemic therapy & $7(22)$ & $7(23)$ \\
\hline Hospitalisation & $11(34)$ & $12(39)$ \\
\hline Disease activity (SCORAD) & $46 \pm 12$ & $46 \pm 19$ \\
\hline Body surface area involved, \% & $41 \pm 24$ & $35 \pm 24$ \\
\hline \multicolumn{3}{|c|}{ Global disease severity assessed by physician (IGA) } \\
\hline Almost clear/mild & $7(23)$ & $7(23)$ \\
\hline Moderate & $14(46)$ & $16(53)$ \\
\hline Severe/very severe & $9(30)$ & $7(23)$ \\
\hline \multicolumn{3}{|c|}{ Global disease severity assessed by patient $(\mathrm{PGA})^{1}$} \\
\hline Almost clear/mild & $11(37)$ & $7(23)$ \\
\hline Moderate & $10(33)$ & $12(40)$ \\
\hline Severe/very severe & $9(30)$ & $11(37)$ \\
\hline \multicolumn{3}{|l|}{ Quality of life impact } \\
\hline (Children's DLQI/DLQI) $^{2}$ & $9 \pm 6$ & $10 \pm 7$ \\
\hline
\end{tabular}

Data are means \pm SD or numbers, with percentages in parentheses. The Children's DLQI was used in children 5-16 years old; the DLQI was used in patients aged 17 years or older; quality of life was not assessed in children aged $<5$ years. None of the observed differences between groups was statistically significant.

${ }^{1} \mathrm{n}=30$ in each group. ${ }^{2} \mathrm{n}=26$ in each group.

[48]. Secondly, physicians in this study regularly applied a validated measurement of disease activity and treatment success, which is not always the case in clinical practice. Additionally, we are not aware of any valid data on the exact amount of medications consumed by patients with moderate-to-severe $\mathrm{AE}$ in clinical practice, but the amounts received by our patients are relatively high.

Surprisingly, both individual response rates and decrease in mean disease activity in patients treated by algorithm therapy were similar to the reported efficacy of systemic treatment modalities including cyclosporine, azathioprine, mycophenolate mofetil and $\gamma$-interferon $[5$, $7,49-52]$. Baseline severity is frequently inflated in RCTs evaluating the efficacy of pharmaceutical interventions 
Table 2. Effectiveness of treatment according to algorithm and treatment as usual

\begin{tabular}{|c|c|c|}
\hline & $\begin{array}{l}\text { Time- and severity-score- } \\
\text { dependent treatment } \\
\text { algorithm }(\mathrm{n}=32)\end{array}$ & $\begin{array}{l}\text { Individualised } \\
\text { symptom-oriented } \\
\text { treatment }(\mathrm{n}=31)\end{array}$ \\
\hline Mean reduction in disease activity (SCORAD) & $21.4[17.6-25.2]$ & $19.3[13.6-24.9]$ \\
\hline \multicolumn{3}{|l|}{ Clinical response (SCORAD $50^{1}$ ) } \\
\hline Proportion with response $\mathrm{e}^{2}$ & $31(97)$ & $27(87)$ \\
\hline Proportion with response at $>50 \%$ of visits ${ }^{3}$ & $14(44)$ & $11(35)$ \\
\hline \multicolumn{3}{|l|}{ Remission (SCORAD $<8$ units) } \\
\hline Proportion with remission ${ }^{2}$ & $9(28)$ & $11(35)$ \\
\hline Proportion with remission at $>50 \%$ of visits ${ }^{3}$ & 0 & 0 \\
\hline \multicolumn{3}{|l|}{ Global disease severity assessed by physician (IGA) } \\
\hline Proportion with improvement ${ }^{2}$ & $31(97)$ & $30(97)$ \\
\hline Proportion with improvement at $>50 \%$ of visits ${ }^{3}$ & $16(50)$ & $17(55)$ \\
\hline \multicolumn{3}{|l|}{ Global disease severity assessed by patient $(\mathrm{PGA})^{4}$} \\
\hline Proportion with improvement ${ }^{2}$ & $28(93)$ & $30(100)$ \\
\hline Proportion with improvement at $>50 \%$ of visits ${ }^{3}$ & $17(57)$ & $17(57)$ \\
\hline Mean improvement in quality of life (DLQI) ${ }^{5}$ & $4.0[1.8-6.2]$ & $4.7[2.7-6.7]$ \\
\hline \multicolumn{3}{|l|}{ Quality of life (DLQI) } \\
\hline Proportion with relevant improvement $(\geq 5 \text { units })^{2}$ & $14(54)$ & $17(65)$ \\
\hline Proportion with relevant improvement ( $\geq 5$ units) at $>50 \%$ of visits ${ }^{3}$ & $37(27)$ & $8(31)$ \\
\hline
\end{tabular}

Data are means, with 95\% CI in square brackets, or numbers, with percentages in parentheses. None of the observed differences between groups was statistically significant.

${ }^{1}$ SCORAD $\leq 50 \%$ of baseline. ${ }^{2}$ At any visit after baseline. ${ }^{3}$ Based on the assumption that the criterion was not met if a study visit was not attended. ${ }^{4} \mathrm{n}=30$ in each group. ${ }^{5} \mathrm{n}=26$ in each group; not assessed in children $<5$ years.

Table 3. Treatment utilisation in patients treated according to evidence-based algorithm compared to treatment as usual stratified by age

\begin{tabular}{|c|c|c|c|c|c|c|}
\hline & \multicolumn{2}{|l|}{ Total } & \multicolumn{2}{|c|}{ Children (aged $2-15$ years) } & \multicolumn{2}{|c|}{ Adults (aged $\geq 16$ years) } \\
\hline & $\begin{array}{l}\text { algorithm } \\
(\mathrm{n}=32)\end{array}$ & $\begin{array}{l}\text { individual } \\
(\mathrm{n}=31)\end{array}$ & $\begin{array}{l}\text { algorithm } \\
(\mathrm{n}=10)\end{array}$ & $\begin{array}{l}\text { individual } \\
(\mathrm{n}=10)\end{array}$ & $\begin{array}{l}\text { algorithm } \\
(\mathrm{n}=22)\end{array}$ & $\begin{array}{l}\text { individual } \\
(\mathrm{n}=21)\end{array}$ \\
\hline \multicolumn{7}{|l|}{ Glucocorticosteroids ${ }^{1}$} \\
\hline Low potency (class I) & 15 & 8 & 6 & 4 & 9 & 4 \\
\hline Intermediate potency (class II) & 28 & 21 & 8 & 9 & 20 & $12^{*}$ \\
\hline High potency (class III) & 14 & $26^{* *}$ & 2 & 6 & 12 & $20^{* *}$ \\
\hline \multicolumn{7}{|l|}{ Calcineurin inhibitors } \\
\hline Pimecrolimus & 30 & $19^{* *}$ & 10 & 8 & 20 & $11^{* *}$ \\
\hline Tacrolimus $0.03 \%$ & 25 & $6^{* *}$ & 8 & 4 & 17 & $2^{* *}$ \\
\hline Tacrolimus $0.1 \%$ & 18 & 21 & 1 & 3 & 17 & 18 \\
\hline Phototherapy & 3 & 1 & - & - & 3 & 1 \\
\hline Prednisolone & 1 & 2 & - & - & 1 & 2 \\
\hline Cyclosporine & 0 & 1 & - & - & 0 & 1 \\
\hline
\end{tabular}

Algorithm = Time- and severity-score-dependent treatment algorithm; individual = individualised symptom-oriented treatment. Data are numbers of patients exposed.

${ }^{*} \mathrm{p}<0.05,{ }^{* *} \mathrm{p}<0.01$; other differences not statistically significant.

${ }^{1}$ Classified according to Niedner [42], very-high-potency (class IV) glucocorticosteroids were not applied in both groups. 
Table 4. Average amount of drugs (g) utilised by treatment according to evidence-based algorithm compared to treatment as usual stratified by age

\begin{tabular}{|c|c|c|c|c|c|c|}
\hline & \multicolumn{2}{|l|}{ Total } & \multicolumn{2}{|c|}{ Children (aged $2-15$ years) } & \multicolumn{2}{|c|}{ Adults (aged $\geq 16$ years) } \\
\hline & $\begin{array}{l}\text { algorithm } \\
(\mathrm{n}=32)\end{array}$ & $\begin{array}{l}\text { individual } \\
(\mathrm{n}=31)\end{array}$ & $\begin{array}{l}\text { algorithm } \\
(\mathrm{n}=10)\end{array}$ & $\begin{array}{l}\text { individual } \\
(\mathrm{n}=10)\end{array}$ & $\begin{array}{l}\text { algorithm } \\
(\mathrm{n}=22)\end{array}$ & $\begin{array}{l}\text { individual } \\
(\mathrm{n}=21)\end{array}$ \\
\hline \multicolumn{7}{|l|}{ Glucocorticosteroids ${ }^{1}$} \\
\hline Low potency (class I) & $36 \pm 53$ & $16 \pm 49$ & $37 \pm 40$ & $36 \pm 80$ & $36 \pm 59$ & $7 \pm 22^{*}$ \\
\hline Intermediate potency (class II) & $211 \pm 328$ & $105 \pm 118$ & $109 \pm 106$ & $68 \pm 56$ & $257 \pm 383$ & $112 \pm 226$ \\
\hline High potency (class III) & $110 \pm 165$ & $247 \pm 271^{*}$ & $53 \pm 129$ & $70 \pm 92$ & $136 \pm 176$ & $331 \pm 289^{* *}$ \\
\hline \multicolumn{7}{|l|}{ Calcineurin inhibitors } \\
\hline Pimecrolimus & $346 \pm 413$ & $134 \pm 164^{* *}$ & $402 \pm 316$ & $186 \pm 150$ & $335 \pm 458$ & $110 \pm 168^{*}$ \\
\hline Tacrolimus $0.03 \%$ & $234 \pm 233$ & $15 \pm 41^{* *}$ & $247 \pm 282$ & $36 \pm 66^{*}$ & $227 \pm 214$ & $4 \pm 14^{* *}$ \\
\hline Tacrolimus $0.1 \%$ & $147 \pm 199$ & $205 \pm 272$ & $24 \pm 76$ & $57 \pm 106$ & $203 \pm 213$ & $276 \pm 299$ \\
\hline
\end{tabular}

Algorithm = Time- and severity-score-dependent treatment algorithm; individual = individualised symptom-oriented treatment. Data are means \pm SD.

${ }^{*} \mathrm{p}<0.05,{ }^{* *} \mathrm{p}<0.01$; other differences not statistically significant.

${ }^{1}$ Classified according to Niedner [42], very-high-potency (class IV) glucocorticosteroids were not applied in both groups.

for AE by including patients only after a wash-out phase for anti-inflammatory drugs $[5,6]$. The fact that there was no wash-out phase in our pragmatic RCT underscores the effectiveness of the tested algorithm. Despite high baseline severity (table 1) only 3 patients allocated to the algorithm group required phototherapy, only a single patient systemic treatment (prednisolone) and none hospitalisation for AE.

At least partly because of the design of the algorithm, highly potent corticosteroids (class III) and calcineurin inhibitors (tacrolimus $0.1 \%$ ) were applied more frequently in the individual group and less potent corticosteroids (classes I and II) and calcineurin inhibitors (pimecrolimus $0.1 \%$ and tacrolimus $0.03 \%$ ) more frequently by algorithm patients. These trends in treatment utilisation were qualitatively similar in children and adults, but less pronounced and not statistically significant in children. Notably, the use of more potent topical treatments in the individual group did not result in better disease control.

The presented data are not sufficient to adequately conduct a cost-effectiveness analysis, but nevertheless they allow some conclusions as to the potential health economic impact of the algorithm approach. The relatively high amounts of medication consumed in both groups should be weighted against presumed savings from avoidance of hospitalisation and systemic treatment. Substantial proportions of patients included in this study had been hospitalised or treated systemically before (table 1).
To closely mirror clinical practice, all patients with moderate-to-severe AE were eligible (except those with prior malignant disease, pregnancy, breastfeeding or without contraception) without requiring a wash-out phase for any drugs administered prior to the study. Participants of our study were assessed over a 12 -month period. This was done to avoid bias by seasonal influences on disease activity and to test the applicability and effectiveness of a treatment algorithm over a meaningful period of time [1].

Although the SCORAD is a well-validated outcome measurement for AE that has been recommended for outcome assessment in clinical trials and everyday practice, it is unknown whether the 8-unit difference we considered as clinically relevant truly is the correct cut-off [8]. If the clinically relevant difference was truly smaller than 8 units, our study would have been underpowered to show superiority of the algorithm approach.

Our study suggests that an evidence-based algorithm for $\mathrm{AE}$ is applicable for children and adults in a dermatological setting: in $93 \%$ of study visits in the algorithm group, physicians actually applied the treatment suggested by the algorithm. However, the algorithm was not superior to individual treatment by dermatologists so that the algorithm approach might not be needed in a dermatological setting. Notably, adequate management of AE does not only require effective anti-inflammatory treatment, but also the management of exacerbating factors and adjunctive treatment with emollients as well as sufficient patient education and instruction $[1,9,19,36,53]$. 
There is increasing evidence that nurse-led clinics for children with AE might be an effective and well-accepted alternative to conventional care from a doctor [53]. Future studies should explore the acceptability, effectiveness, efficiency and ease of use of an algorithm-based treatment of $\mathrm{AE}$ in a large cohort of patients by means of a multi-disciplinary study including paediatricians, dermatologists, general practitioners and nurse-led clinics.

\section{Acknowledgements}

We thank K. Blümlein for assistance in study coordination, $\mathrm{K}$. Wirth for monitoring, and our colleagues from the Department of Dermatology, Technical University Dresden, Germany, for their role in the recruitment and treatment of patients.

\section{References}

1 Williams HC: Clinical practice: atopic dermatitis. N Engl J Med 2005;352:2314-2324.

2 International Study of Asthma and Allergies in Childhood (ISAAC) Steering Committee: Worldwide variation in prevalence of symptoms of asthma, allergic rhinoconjunctivitis, and atopic eczema: ISAAC. Lancet 1998;351: $1225-1232$.

3 Chamlin SL, Frieden IJ, Williams ML, Chren MM: Effects of atopic dermatitis on young American children and their families. Pediatrics 2004;114:607-611.

4 Carroll CL, Balkrishnan R, Feldman SR, Fleischer AB Jr, Manuel JC: The burden of atopic dermatitis: impact on the patient, family, and society. Pediatr Dermatol 2005; 22:192-199.

5 Schmitt J, Schakel K, Schmitt N, Meurer M: Systemic treatment of severe atopic eczema: a systematic review. Acta Derm Venereol 2007;87:100-111.

6 Hoare C, Li Wan Po A, Williams H: Systematic review of treatments for atopic eczema. Health Technol Assess 2000;4:1-191.

7 Schmitt J, Schmitt NM, Meurer M: Cyclosporin in the treatment of patients with atopic eczema - a systematic review and metaanalysis. J Eur Acad Dermatol Venereol 2007;21:606-619.

8 Schmitt J, Langan SM, Williams HC: What are the best outcome measurements for atopic eczema? A systematic review. J Allergy Clin Immunol 2007;120:1389-1398.

9 Ellis C, Luger T, Abeck D, Allen R, GrahamBrown RA, De Prost Y, Eichenfield LF, Ferrandiz C, Giannetti A, Hanifin J, Koo JY, Leung D, Lynde C, Ring J, Ruiz-Maldonado $\mathrm{R}$, Saurat JH: International Consensus Conference on Atopic Dermatitis II (ICCAD II): clinical update and current treatment strategies. Br J Dermatol 2003;148(suppl 63):3-10.

10 Hanifin JM, Cooper KD, Ho VC, Kang S, Krafchik BR, Margolis DJ, Schachner LA, Sidbury R, Whitmore SE, Sieck CK, Van Voorhees AS: Guidelines of care for atopic dermatitis, developed in accordance with the American Academy of Dermatology (AAD)/ American Academy of Dermatology Association 'Administrative Regulations for Evidence-Based Clinical Practice Guidelines'. J Am Acad Dermatol 2004;50:391-404.
11 McHenry PM, Williams HC, Bingham EA: Management of atopic eczema. Joint Workshop of the British Association of Dermatologists and the Research Unit of the Royal College of Physicians of London. BMJ 1995 310:843-847.

12 Expert Panel Report 3 (EPR-3): Guidelines for the Diagnosis and Management of Asthma - Summary Report 2007. J Allergy Clin Immunol 2007;120:S94-S138

13 Adli M, Berghofer A, Linden M, Helmchen $\mathrm{H}$, Muller-Oerlinghausen B, Mackert A, Stamm T, Bauer M: Effectiveness and feasibility of a standardized stepwise drug treatment regimen algorithm for inpatients with depressive disorders: results of a 2-year observational algorithm study. J Clin Psychiatry 2002;63:782-790.

14 Zech DF, Grond S, Lynch J, Hertel D, Lehmann KA: Validation of World Health Organization Guidelines for cancer pain relief: a 10-year prospective study. Pain 1995;63:6576

15 Illi S, von Mutius E, Lau S, Nickel R, Gruber C, Niggemann B, Wahn U: The natural course of atopic dermatitis from birth to age 7 years and the association with asthma. J Allergy Clin Immunol 2004;113:925-931.

16 Spergel JM, Paller AS: Atopic dermatitis and the atopic march. J Allergy Clin Immunol 2003;112:S118-S127.

17 Williams H, Wüthrich B: The natural history of atopic dermatitis; in Williams $\mathrm{H}$ (ed): Atopic Dermatitis: The Epidemiology, Causes, and Prevention of Atopic Eczema. Cambridge, Cambridge University Press, 2000, pp 41-59.

18 Lammintausta K, Kalimo K, Raitala R, For sten Y: Prognosis of atopic dermatitis: a prospective study in early adulthood. Int J Dermatol 1991;30:563-568.

19 Leung DY, Bieber T: Atopic dermatitis. Lancet 2003;361:151-160.

20 Thestrup-Pedersen K: Treatment principles of atopic dermatitis. J Eur Acad Dermatol Venereol 2002;16:1-9.

21 Abeck D, Strom K: Optimal management of atopic dermatitis. Am J Clin Dermatol 2000 1:41-46.
22 Aliaga A, Rodriguez M, Armijo M, Bravo J, Avila AL, Mascaro JM, Ferrando J, Del Rio R, Lozano R, Balaguer A: Double-blind study of prednicarbate versus fluocortin butyl ester in atopic dermatitis. Int J Dermatol 1996; 35:131-132.

23 Thomas KS, Armstrong S, Avery A, Po AL, O'Neill C, Young S, Williams HC: Randomised controlled trial of short bursts of a potent topical corticosteroid versus prolonged use of a mild preparation for children with mild or moderate atopic eczema. BMJ 2002;324:768

24 Wolkerstorfer A, Strobos MA, Glazenburg EJ, Mulder PG, Oranje AP: Fluticasone propionate $0.05 \%$ cream once daily versus clobetasone butyrate $0.05 \%$ cream twice daily in children with atopic dermatitis. J Am Acad Dermatol 1998;39:226-231.

25 Reitamo S, Rustin M, Ruzicka T, Cambazard F, Kalimo K, Friedmann PS, Schoepf E, Lahfa M, Diepgen TL, Judodihardjo H, Wollenberg A, Berth-Jones J, Bieber T: Efficacy and safety of tacrolimus ointment compared with that of hydrocortisone butyrate ointment in adult patients with atopic dermatitis. J Allergy Clin Immunol 2002;109:547-555.

26 Reitamo S, Van Leent EJ, Ho V, Harper J, Ruzicka T, Kalimo K, Cambazard F, Rustin M, Taieb A, Gratton D, Sauder D, Sharpe G, Smith C, Junger M, de Prost Y: Efficacy and safety of tacrolimus ointment compared with that of hydrocortisone acetate ointment in children with atopic dermatitis. J Allergy Clin Immunol 2002;109:539-546.

27 Granlund H, Erkko P, Remitz A, Langeland T, Helsing P, Nuutinen M, Reitamo S: Comparison of cyclosporin and UVAB phototherapy for intermittent one-year treatment of atopic dermatitis. Acta Derm Venereol 2001;81:22-27.

28 Luger T, Van Leent EJ, Graeber M, Hedgecock S, Thurston M, Kandra A, Berth-Jones J, Bjerke J, Christophers E, Knop J, Knulst AC, Morren M, Morris A, Reitamo S, RoedPetersen J, Schoepf E, Thestrup-Pedersen K, Van der Valk PG, Bos JD: SDZ ASM 981: an emerging safe and effective treatment for atopic dermatitis. Br J Dermatol 2001;144: 788-794. 
29 Charman C, Chambers C, Williams H: Measuring atopic dermatitis severity in randomized controlled clinical trials: what exactly are we measuring? J Invest Dermatol 2003; 120:932-941.

30 Charman C, Williams H: Outcome measures of disease severity in atopic eczema. Arch Dermatol 2000;136:763-769.

31 Hanifin J, Rajka G: Diagnostic features of atopic dermatitis. Acta Derm Venereol (Stockh) 1980;92(suppl):44-47.

32 Severity scoring of atopic dermatitis: the SCORAD index. Consensus Report of the European Task Force on Atopic Dermatitis. Dermatology 1993;186:23-31.

33 Schachner LA, Lamerson C, Sheehan MP, Boguniewicz M, Mosser J, Raimer S, Shull T, Jaracz E: Tacrolimus ointment $0.03 \%$ is safe and effective for the treatment of mild to moderate atopic dermatitis in pediatric patients: results from a randomized, doubleblind, vehicle-controlled study. Pediatrics 2005; 116:e334-e342.

34 Lewis-Jones MS, Finlay AY: The Children's Dermatology Life Quality Index (CDLQI): initial validation and practical use. Br J Dermatol 1995;132:942-949.

35 Finlay AY, Khan GK: Dermatology Life Quality Index (DLQI) - a simple practical measure for routine clinical use. Clin Exp Dermatol 1994;19:210-216.

36 Staab D, Diepgen TL, Fartasch M, Kupfer J, Lob-Corzilius T, Ring J, Scheewe S, Scheidt R, Schmid-Ott G, Schnopp C, Szczepanski R, Werfel T, Wittenmeier M, Wahn U, Gieler $\mathrm{U}$ : Age related, structured educational programmes for the management of atopic dermatitis in children and adolescents: multicentre, randomised controlled trial. BMJ 2006;332:933-938.
37 Kunz B, Oranje AP, Labreze L, Stalder JF, Ring J, Taieb A: Clinical validation and guidelines for the SCORAD index: consensus report of the European Task Force on Atopic Dermatitis. Dermatology 1997;195: 10-19.

38 Pucci N, Novembre E, Cammarata MG, Bernardini R, Monaco MG, Calogero C, Vierucci A: Scoring atopic dermatitis in infants and young children: distinctive features of the SCORAD index. Allergy 2005;60:113-116.

39 Lewis V, Finlay AY: 10 years experience of the Dermatology Life Quality Index (DLQI). J Invest Dermatol Symp Proc 2004;9:169180.

40 Khilij FA, Gonzalez M, Finlay AY: Clinical meaning of change in Dermatology Life Quality Index scores. Br J Dermatol 2002; 147(suppl 62):50.

41 Common Terminology Criteria for Adverse Events v3.0 (CTCAE). Rockville, DTCD, NCI, NIH, DHHS, 2003.

42 Niedner R: External administration of glucocorticosteroids. 1. Administration guidelines - classification. Fortschr Med 1992;110: 327-329.

43 Rosner B: Fundamentals of Biostatistics. Pacific Grove, Duxbury, 2000.

44 Patel TS, Greer SC, Skinner RB Jr: Cancer concerns with topical immunomodulators in atopic dermatitis: overview of data and recommendations to clinicians. Am J Clin Dermatol 2007;8:189-194.

45 Thaci D, Salgo R: The topical calcineurin inhibitor pimecrolimus in atopic dermatitis: a safety update. Acta Dermatovenereol Alp Panonica Adriat 2007; 16:60-62.

46 Margolis DJ, Hoffstad O, Bilker W: Lack of association between exposure to topical calcineurin inhibitors and skin cancer in adults. Dermatology 2007;214:289-295.
47 Ashcroft DM, Dimmock P, Garside R, Stein K, Williams HC: Efficacy and tolerability of topical pimecrolimus and tacrolimus in the treatment of atopic dermatitis: meta-analysis of randomised controlled trials. BMJ 2005;330:516.

48 Ohya Y, Williams H, Steptoe A, Saito H, Iikura Y, Anderson R, Akasawa A: Psychosocial factors and adherence to treatment advice in childhood atopic dermatitis. J Invest Dermatol 2001;117:852-857.

49 Meggitt SJ, Gray JC, Reynolds NJ: Azathioprine dosed by thiopurine methyltransferase activity for moderate-to-severe atopic eczema: a double-blind, randomised controlled trial. Lancet 2006;367:839-846.

50 Sowden JM, Berth-Jones J, Ross JS, Motley RJ, Marks R, Finlay AY, Salek MS, GrahamBrown RA, Allen BR, Camp RD: Doubleblind, controlled, crossover study of cyclosporin in adults with severe refractory atopic dermatitis. Lancet 1991;338:137-140.

51 Grundmann-Kollmann M, Podda M, Ochsendorf F, Boehncke WH, Kaufmann R, Zollner TM: Mycophenolate mofetil is effective in the treatment of atopic dermatitis. Arch Dermatol 2001;137:870-873.

52 Hanifin JM, Schneider LC, Leung DY, Ellis CN, Jaffe HS, Izu AE, Bucalo LR, Hirabayashi SE, Tofte SJ, Cantu-Gonzales G: Recombinant interferon gamma therapy for atopic dermatitis. J Am Acad Dermatol 1993;28: 189-197.

53 Moore E, Williams A, Manias E, Varigos G: Nurse-led clinics reduce severity of childhood atopic eczema: a review of the literature. Br J Dermatol 2006;155:1242-1248. 